\title{
Subharmonic Solutions of Nonautonomous Second Order Differential Equations with Singular Nonlinearities
}

\author{
N. Daoudi-Merzagui, ${ }^{1}$ F. Derrab, ${ }^{2}$ and A. Boucherif ${ }^{3}$ \\ ${ }^{1}$ Department of Mathematics, University of Tlemcen, Tlemcen 13000, Algeria \\ ${ }^{2}$ Department of Mathematics, University of Sidi Bel Abbes, Sidi Bel Abbes 22000, Algeria \\ ${ }^{3}$ Department of Mathematics and Statistics, King Fahd University of Petroleum and Minerals, \\ P.O. Box 5046, Dhahran 31261, Saudi Arabia
}

Correspondence should be addressed to A. Boucherif, aboucher@kfupm.edu.sa

Received 4 November 2011; Revised 14 January 2012; Accepted 19 January 2012

Academic Editor: Juan J. Nieto

Copyright (C) 2012 N. Daoudi-Merzagui et al. This is an open access article distributed under the Creative Commons Attribution License, which permits unrestricted use, distribution, and reproduction in any medium, provided the original work is properly cited.

\begin{abstract}
We discuss the existence of subharmonic solutions for nonautonomous second order differential equations with singular nonlinearities. Simple sufficient conditions are provided enable us to obtain infinitely many distinct subharmonic solutions. Our approach is based on a variational method, in particular the saddle point theorem.
\end{abstract}

\section{Introduction and Main Result}

In this paper we discuss the problem of the existence of infinitely many subharmonic solutions for nonautonomous second order differential equations with singular nonlinearities of the form

$$
u^{\prime \prime}(t)+f(t, u(t))=e(t)
$$

where $f: \mathbb{R}^{2} \rightarrow \mathbb{R}$ is continuous, is $T$-periodic, in its first argument with $T>0$, and presents a singularity with respect to its second argument. Here by a subharmonic solution we mean a $k T$-periodic solution for any integer $k$ if $T>0$ is the minimal period. When the solution is not $T$-periodic we call it a true subharmonic. It was pointed out in [1] that singular differential equations of the form (1.1) appear in the description of many phenomena in the applied sciences, such as the Brillouin focusing system and nonlinear elasticity. Several authors have 
investigated the problem of existence of periodic solutions for second order differential equations with singular nonlinearities (see [2-4] and the references therein). Topological and variational methods are the two main techniques that have been developed for the study of (1.1). We refer the interested reader to the paper [1] for details and references on the topological methods. In this work we shall rely on the saddle point theorem, see $[5,6]$, to prove our main result. We use the truncation techniques introduced in [7] to modify our problem to one without singularities. We assume that the nonlinearity $f$ is monotone with respect to its first variable $t$. When $f$ is increasing, our result generalizes the result in [8]. We can obtain the same result by considering the monotonicity of the potential function instead of the field $f$. For more results on the subject and different techniques one can consult the papers [9-12]. We should point out some related recent articles, for instance $[13,14]$.

Throughout this paper we shall use the following notations. Let $I=[0, T] . L^{p}(I)$ is the classical Lebesgue space of functions $u: I \rightarrow \mathbb{R}$ such that $|u(\cdot)|^{p}$ is integrable, and for $u \in$ $L^{p}(I)$ we define its norm by

$$
\|u\|_{L^{p}}=\left(\int_{0}^{T}|u(t)|^{p} d t\right)^{1 / p} .
$$

Let $\|u\|_{\infty}=\sup \{|u(t)| ; t \in[0, k T]\}$. For $T>0$ and $k \in \mathbb{N}$ we let $H_{k T}^{1}=\{u \in$ $\left.W^{1,2}([0, k T], \mathbb{R}) ; u(0)=u(k T)\right\}$ and for $u \in H_{k T}^{1}$ we define its norm by

$$
\|u\|_{H_{k T}^{1}}=\left(\|u\|_{L^{2}}+\left\|u^{\prime}\right\|_{L^{2}}\right)^{1 / 2}
$$

$H_{k T}^{1}$ endowed with the norm $\|\cdot\|_{H_{k T}^{1}}$ is a reflexive Banach space. Also $H_{k T}^{1}=H^{+} \oplus H^{-}$, orthogonal decomposition, where $H^{+}$is the subspace of constant functions in $H_{k T}^{1}$ and $H^{-}$ denotes the subspace of functions in $H_{k T}^{1}$ with mean value zero; so that $u \in H_{k T}^{1}$ can be written as $u=\bar{u}+\tilde{u}$ with $\bar{u} \in H^{+}$and $\tilde{u} \in H^{-}$.

We shall assume that $e: \mathbb{R} \rightarrow \mathbb{R}$ is a locally integrable $T$-periodic function. We denote the mean value of $e$ by $\bar{e}$, that is, $\bar{e}=1 / T \int_{0}^{T} e(t) d t$. It follows that $\bar{e}_{,}\|e\|_{L^{1}}$ and $\|e\|_{L^{2}}$ are bounded. Moreover, since $e$ is $T$-periodic, we have $\int_{0}^{k T} e(t) d t=\sum_{j=0}^{k-1} \int_{0}^{T} e(t+j T) d t=$ $\sum_{j=0}^{k-1} \int_{0}^{T} e(t) d t=k \int_{0}^{T} e(t) d t=k T \bar{e}$.

Let $F(t, u)=\int_{1}^{u} f(t, s) d s$ be an antiderivative of $f$ defined for all $u \in \mathbb{R}$ and for all $t \in I$.

We introduce the following assumptions on the nonlinearity.

(H1) $f: \mathbb{R} \times(0,+\infty) \rightarrow \mathbb{R}$ is continuous, $f(t+T, u)=f(t, u)$ for all $(t, u) \in \mathbb{R} \times(0,+\infty)$, and such that

(i) $t \rightarrow f(t, u)$ is monotone for each fixed $u$ in $(0,+\infty)$,

(ii) $\lim _{u \rightarrow 0^{+}} f(t, u)=-\infty$, uniformly in $t \in I$,

(iii) $f(0, u)=f(T, u)>\bar{e}$ for all $u>1$.

$(\mathrm{H} 2)$

(i) $\lim _{u \rightarrow+\infty} 2 F(t, u) / u^{2}=0$, uniformly in $t \in I$.

(ii) $\lim _{u \rightarrow+\infty} \int_{0}^{T}[F(t, u)-\bar{e} u] d t=+\infty$. 
Theorem 1.1. Assume that e is a locally integrable T-periodic function. If (H1) and (H2) are satisfied then (1.1) has a sequence $\left(u_{k}\right)_{k \geq 1}$ of $k T$-periodic solutions whose amplitudes and minimal periods tend to infinity. In particular, if $T$ is the minimal period of $e$ and of $f$ with respect to $t,(1.1)$ admits solutions with minimal periods $k T$ for every sufficiently Large Integer $k$.

\section{Proof of Theorem 1.1}

The proof of this result will be based on several auxiliary results.

\subsection{Modification of the Problem}

Define the truncation function $f_{r}: \mathbb{R}^{2} \rightarrow \mathbb{R}, 0<r \leq 1$, by

$$
f_{r}(t, u)= \begin{cases}f(t, u), & u \geq r \\ f(t, r), & u<r .\end{cases}
$$

Note that condition (H1) implies that $f_{r}$ is continuous with respect to $(t, u) \in I \times \mathbb{R}$ and $T$-periodic with respect to its first variable $t$.

Lemma 2.1. Assume (H1) and (H2) (ii) are satisfied. Then there exists $d>1$ such that for every $u \in(0,1 / d) \cup(d,+\infty)$

$$
(f(t, u)-\bar{e})(u-1)>0, \quad \text { uniformly in } t \in I .
$$

Proof. First, it follows from (H1)(ii) that for any $A>0$, there is $\delta_{A}>0$ such that for every $u \in\left(0, \delta_{A}\right)$ we have $f(t, u)<-A$, uniformly in $t \in I$.

In particular for $A>2|\bar{e}|+1$, there is $\delta_{A}>0$ such that for every $u \in\left(0, \delta_{A}\right)$ it holds

$$
f(t, u)-\bar{e}<-|\bar{e}|-1<0 .
$$

Choose $d_{1}>1$ such that $1 / d_{1}<\delta_{A}$. Then for every $u \in\left(0,1 / d_{1}\right)$, we have $f(t, u)-\bar{e}<0$.

Therefore, condition (H1)(ii) implies that there exists $d_{1}>1$ such that for every $u \in$ $\left(0,1 / d_{1}\right)$ it holds $(f(t, u)-\bar{e})(u-1)>0$, uniformly in $t \in I$.

Next, condition (H2) (ii) implies that for any $B>1$, sufficiently large, there is $\chi_{B}>0$, large enough, such that for every $u>\chi_{B}$ we have

$$
\int_{0}^{T}[F(t, u)-\bar{e} u] d t>B
$$

Hence, there exists $d_{2}$, sufficiently large such that $d_{2}>\max \left(1, X_{B}\right)$ and for every $u>d_{2}$ it holds

$$
\int_{0}^{T}[F(t, u)-\bar{e} u] d t>B
$$

We show that for $u>d_{2}$ we have $f(t, u)-\bar{e}>0$, uniformly in $t \in I$. Assume, on the contrary, that there exists $y>d_{2}$ for which $f\left(t_{0}, y\right)-\bar{e} \leq 0$, for some $t_{0} \in(0, T)$. By the continuity of 
$f$ on $I$ and $\left((H 1)(\right.$ iii) $)$, there exists $\sigma \in(0, T)$ such that $f(\sigma, u)=\max _{t \in I} f(t, u)$. If $t_{0} \leq \sigma$ then $0<f(0, y)-\bar{e}<f\left(t_{0}, y\right)-\bar{e} \leq 0$, and this is a contradiction. Similarly, if $t_{0} \geq \sigma$ then $0<$ $f(0, y)-\bar{e}=f(T, y)-\bar{e}<f\left(t_{0}, y\right)-\bar{e} \leq 0$, and we again arrive at a contradiction.

Hence we deduce that if (H1) and (H2) (ii) hold, then there exists $d>\max \left(d_{1}, d_{2}\right)$ such that for any $u \in(0,1 / d) \cup(d,+\infty)(f(t, u)-\bar{e})(u-1)>0$, uniformly in $t \in I$. This completes the proof of Lemma 2.1.

Lemma 2.2. For every positive integer $k$, there exist $r_{k}$ and $R_{k}$ with $0<r_{k}<1 / d<d<R_{k}$ such that for any $\mu \in\left(0, r_{k}\right]$ each $k T$-periodic solution $u$ of

$$
u^{\prime \prime}(t)+f_{\mu}(t, u(t))=e(t)
$$

satisfies

$$
r_{k} \leq u(t) \leq R_{k}, \quad \forall t \in \mathbb{R}
$$

In particular, any $k T$-periodic solution of (2.6), with $\mu=r_{k}$ is a solution of (1.1).

Proof. This is essentially Proposition 2.1 in [8]. We shall use some ideas from [8] (see also [15]). Fix $k \in \mathbb{N}$, and suppose, on the contrary, that for each integer $n$, there exist $\mu_{n} \in(0,1 / n)$ and a $k T$-periodic solution $u_{n}$ satisfying

$$
u_{n}^{\prime \prime}(t)+f_{\mu_{n}}\left(t, u_{n}(t)\right)=e(t)
$$

and $\left\{u_{n}(t) ; t \in \mathbb{R}\right\} \nsubseteq[1 / n, n]$.

Claim 1. Let $d$ be as in Lemma 2.1 and let $u_{n}$ be as above. Then for every $n$ there exists $\tau_{n} \in$ $[0, k T]$ such that $u_{n}\left(\tau_{n}\right) \in[1 / d, d]$.

Indeed, it follows from (2.8) that

$$
\int_{0}^{k T} f_{\mu_{n}}\left(t, u_{n}(t)\right) d t=k T \bar{e}
$$

Now, if $u_{n}(t)>d$ for all $t \in[0, k T]$, then Lemma 2.1 implies that $f_{\mu_{n}}\left(t, u_{n}(t)\right)-\bar{e}>0$, which in turn yields $\int_{0}^{k T} f_{\mu_{n}}\left(t, u_{n}(t)\right) d t>k T \bar{e}$.

This contradicts (2.9). On the other hand, if $u_{n}(t)<1 / d$ for all $t \in[0, k T]$, then $f_{\mu_{n}}\left(t, u_{n}(t)\right)-\bar{e}<0$, so that $\int_{0}^{k T} f_{\mu_{n}}\left(t, u_{n}(t)\right) d t<k T \bar{e}$. This is again a contradiction to (2.9).

Claim 2. There exists $R>0$ such that $M_{n}=\max _{t \in[0, k T]} u_{n}(t) \leq R$ for each integer $n$. To prove the claim notice, there exists $t_{n}^{1} \in[0, k T]$ such that $u_{n}\left(t_{n}^{1}\right)=M_{n}$. If $u_{n}\left(t_{n}^{1}\right)=M_{n} \in[1 / d, d]$ then $u_{n}(t) \leq R$ for any $R>d$. So, assume that there exists a subsequence of $\left(u_{n}\right)_{n}$, which we label 
the same, for which $M_{n} \rightarrow+\infty$ when $n \rightarrow+\infty$. So that $M_{n}>d$ for $n$ large enough. Since $u_{n}\left(\tau_{n}\right)<d$, there exists an interval $\left[\alpha_{n}, \beta_{n}\right]$, containing $t_{n}^{1}$, such that

$$
\begin{gathered}
\beta_{n}-\alpha_{n} \leq k T, \\
u_{n}\left(\alpha_{n}\right)=d=u_{n}\left(\beta_{n}\right), \\
d \leq u_{n}(t) \leq u_{n}\left(t_{n}^{1}\right), \text { for all } t \in\left[\alpha_{n}, \beta_{n}\right] .
\end{gathered}
$$

Equation (2.8) can be written as

$$
\begin{gathered}
u_{n}^{\prime}(t)=v_{n}(t)+\int_{\alpha_{n}}^{t}[e(s)-\bar{e}] d s, \\
v_{n}^{\prime}(t)=-f_{\mu_{n}}\left(t, u_{n}(t)\right)+\bar{e} .
\end{gathered}
$$

Since for all $t \in\left(\alpha_{n}, \beta_{n}\right), u_{n}(t)>d$ and $\mu_{n}<1 / n$, then the second equation in (2.11) is equivalent to

$$
v_{n}^{\prime}(t)=-f\left(t, u_{n}(t)\right)+\bar{e}
$$

Lemma 2.1 implies that $f\left(t, u_{n}(t)\right)-\bar{e}>0$ for all $t \in\left[\alpha_{n}, \beta_{n}\right]$. Then $v_{n}^{\prime}(t)<0$ for all $t \in\left[\alpha_{n}, \beta_{n}\right]$ and hence $v_{n}$ is decreasing on $\left[\alpha_{n}, \beta_{n}\right]$. The first equation in (2.11) implies

$$
u_{n}^{\prime}(t) \leq v_{n}\left(\alpha_{n}\right)+\int_{\alpha_{n}}^{t}[e(s)-\bar{e}] d s \quad \forall t \in\left[\alpha_{n}, \beta_{n}\right] .
$$

This yields

$$
u_{n}^{\prime}(t) \leq v_{n}\left(\alpha_{n}\right)+\|e\|_{L^{1}}+k T|\bar{e}| \quad \forall t \in\left[\alpha_{n}, \beta_{n}\right]
$$

Integrating the above inequality over $\left[\alpha_{n}, t_{n}^{1}\right] \subset\left[\alpha_{n}, \beta_{n}\right]$ we obtain

$$
u_{n}\left(t_{n}^{1}\right)-u_{n}\left(\alpha_{n}\right)=M_{n}-d \leq k T\left[v_{n}\left(\alpha_{n}\right)+\|e\|_{L^{1}}+k T|\bar{e}|\right] .
$$

Equation (2.15) leads to

$$
\lim _{n \rightarrow+\infty}\left(1-\frac{d}{M_{n}}\right) \leq k T \lim _{n \rightarrow+\infty}\left(\frac{v_{n}\left(\alpha_{n}\right)+\|e\|_{L^{1}}+k T|\bar{e}|}{M_{n}}\right) .
$$

Hence

$$
1 \leq k T \lim _{n \rightarrow+\infty} \frac{v_{n}\left(\alpha_{n}\right)}{M_{n}}
$$

It is clear from (2.17) that $v_{n}\left(\alpha_{n}\right) \rightarrow+\infty$ when $n \rightarrow+\infty$. So that, for $n$ large enough, we have

$$
v_{n}\left(\alpha_{n}\right) \geq\|e\|_{L^{1}}+k T|\bar{e}| .
$$


Since $M_{n}=u_{n}\left(t_{n}^{1}\right)$, we have $u_{n}^{\prime}\left(t_{n}^{1}\right)=0$. It follows from the first equation in (2.11) that

$$
\left|v_{n}\left(t_{n}^{1}\right)\right|=\left|\int_{\alpha_{n}}^{t_{n}^{1}}[e(s)-\bar{e}] d s\right| \leq\|e\|_{L^{1}}+k T|\bar{e}| .
$$

We see from (2.18) and (2.19) that for $n$ large enough

$$
v_{n}\left(\alpha_{n}\right) \geq\|e\|_{L^{1}}+k T|\bar{e}| \geq v_{n}\left(t_{n}^{1}\right) .
$$

Since $v_{n}(\cdot)$ is continuous on $\left[\alpha_{n}, \beta_{n}\right]$, then, for $n$ large enough, there exists at least one $t_{n}^{*} \in$ $\left(\alpha_{n}, t_{n}^{1}\right)$ such that $v_{n}\left(t_{n}^{*}\right)=\|e\|_{L^{1}}+k T|\bar{e}|$. We denote by $t_{n}^{2}$, the first such $t_{n}^{*}$. Then

$$
v_{n}\left(t_{n}^{2}\right)=\|e\|_{L^{1}}+k T|\bar{e}|
$$

We distinguish two cases.

Case 1. $f(\cdot, s)$ is increasing for each fixed $s \in(0,+\infty)$.

Consider the function $B_{1}$ defined by

$$
B_{1}(t)=F\left(t_{n}^{2}, u_{n}(t)\right)-\bar{e} u_{n}(t)+\frac{1}{2}\left(v_{n}(t)-\|e\|_{L^{1}}-k T|\bar{e}|\right)^{2} .
$$

Then

$$
B_{1}^{\prime}(t)=\left(f\left(t_{n}^{2}, u_{n}(t)\right)-\bar{e}\right) u_{n}^{\prime}(t)+\left(v_{n}(t)-\|e\|_{L^{1}}-k T|\bar{e}|\right) v_{n}^{\prime}(t) .
$$

Since $v_{n}^{\prime}(t)=-f\left(t, u_{n}(t)\right)+\bar{e}$, it follows from the first equation in (2.11)

$$
\begin{aligned}
B_{1}^{\prime}(t)= & \left(f\left(t_{n}^{2}, u_{n}(t)\right)-\bar{e}\right)\left(v_{n}(t)+\int_{\alpha_{n}}^{t}[e(s)-\bar{e}] d s\right) \\
& +\left(v_{n}(t)-\|e\|_{L^{1}}-k T|\bar{e}|\right)\left(-f\left(t, u_{n}(t)\right)+\bar{e}\right) \\
= & \left(f\left(t_{n}^{2}, u_{n}(t)\right)-\bar{e}\right)\left(v_{n}(t)+\int_{\alpha_{n}}^{t}[e(s)-\bar{e}] d s+\|e\|_{L^{1}}+k T|\bar{e}|-\|e\|_{L^{1}}-k T|\bar{e}|\right) \\
& +\left(v_{n}(t)-\|e\|_{L^{1}}-k T|\bar{e}|\right)\left(-f\left(t, u_{n}(t)\right)+\bar{e}\right) .
\end{aligned}
$$

Hence

$$
\begin{aligned}
B_{1}^{\prime}(t)= & \left(f\left(t_{n}^{2}, u_{n}(t)\right)-\bar{e}\right)\left(\int_{\alpha_{n}}^{t}[e(s)-\bar{e}] d s+\|e\|_{L^{1}}+k T|\bar{e}|\right) \\
& +\left(f\left(t_{n}^{2}, u_{n}(t)\right)-f\left(t, u_{n}(t)\right)\right)\left(v_{n}(t)-\|e\|_{L^{1}}-k T|\bar{e}|\right) .
\end{aligned}
$$


Since, for all $t \in\left[\alpha_{n}, \beta_{n}\right]$

$$
\left|\int_{\alpha_{n}}^{t}[e(s)-\bar{e}] d s\right| \leq\|e\|_{L^{1}}+k T|\bar{e}|
$$

it follows that for all $t \in\left[\alpha_{n}, \beta_{n}\right]$

$$
\int_{\alpha_{n}}^{t}[e(s)-\bar{e}] d s+\|e\|_{L^{1}}+k T|\bar{e}| \geq 0
$$

Also, Lemma 2.1 implies that $f\left(t_{n}^{2}, u_{n}(t)\right)-\bar{e}>0$ for all $t \in\left[\alpha_{n}, \beta_{n}\right]$. Furthermore, the monotonicity of $f$ implies that $f\left(t_{n}^{2}, u_{n}(t)\right)-f\left(t, u_{n}(t)\right) \geq 0$ for all $t \in\left[\alpha_{n}, t_{n}^{2}\right]$. Since $v_{n}(\cdot)$ is decreasing on $\left[\alpha_{n}, t_{n}^{2}\right]$ and $v_{n}\left(t_{n}^{2}\right)=\|e\|_{L^{1}}+k T|\bar{e}|$, it follows that

$$
v_{n}(t)-\|e\|_{L^{1}}-k T|\bar{e}| \geq v_{n}\left(t_{n}^{2}\right)-\|e\|_{L^{1}}-k T|\bar{e}|=0
$$

for all $t \in\left[\alpha_{n}, t_{n}^{2}\right]$. Now, Lemma 2.1 combined with (2.27), (2.28), and the monotonicity of $f$ with respect to its first variable shows that

$$
B_{1}^{\prime}(t) \geq 0, \quad \forall t \in\left[\alpha_{n}, t_{n}^{2}\right]
$$

Thus, the function $B_{1}$ is increasing on $\left[\alpha_{n}, t_{n}^{2}\right]$. Since $u_{n}\left(\alpha_{n}\right)=d$,

$$
\begin{aligned}
B_{1}\left(\alpha_{n}\right) & =F\left(t_{n}^{2}, d\right)-\bar{e} d+\frac{1}{2}\left(v_{n}\left(\alpha_{n}\right)-\|e\|_{L^{1}}-k T|\bar{e}|\right)^{2} \\
& \leq B_{1}\left(t_{n}^{2}\right)=F\left(t_{n}^{2}, u_{n}\left(t_{n}^{2}\right)\right)-\bar{e} u_{n}\left(t_{n}^{2}\right)+\frac{1}{2}\left(v_{n}\left(t_{n}^{2}\right)-\|e\|_{L^{1}}-k T|\bar{e}|\right)^{2} .
\end{aligned}
$$

Since $v_{n}\left(t_{n}^{2}\right)-\|e\|_{L^{1}}-k T|\bar{e}|=0$, it follows that

$$
B_{1}\left(\alpha_{n}\right)=F\left(t_{n}^{2}, d\right)-\bar{e} d \leq F\left(t_{n}^{2}, u_{n}\left(t_{n}^{2}\right)\right)-\bar{e} u_{n}\left(t_{n}^{2}\right)
$$

Notice that

$$
F\left(t_{n}^{2}, u_{n}\left(t_{n}^{2}\right)\right)-\bar{e} u_{n}\left(t_{n}^{2}\right)=\int_{1}^{d}\left(f\left(t_{n}^{2}, s\right)-\bar{e}\right) d s+\int_{d}^{u_{n}\left(t_{n}^{2}\right)}\left(f\left(t_{n}^{2}, s\right)-\bar{e}\right) d s-\bar{e}
$$

Also, $f\left(t_{n}^{2}, s\right)-\bar{e}>0$ if $s \in\left(d, u_{n}\left(t_{n}^{2}\right)\right] \subset\left[d, u_{n}\left(t_{n}^{1}\right)\right]$ (see Lemma 2.1). It follows that

$$
\int_{d}^{u_{n}\left(t_{n}^{2}\right)}\left[f\left(t_{n}^{2}, s\right)-\bar{e}\right] d s \leq \int_{d}^{u_{n}\left(t_{n}^{1}\right)}\left[f\left(t_{n}^{2}, s\right)-\bar{e}\right] d s
$$


Hence

$$
B_{1}\left(\alpha_{n}\right) \leq \int_{1}^{d}\left[f\left(t_{n}^{2}, s\right)-\bar{e}\right] d s+\int_{d}^{u_{n}\left(t_{n}^{1}\right)}\left[f\left(t_{n}^{2}, s\right)-\bar{e}\right] d s-\bar{e}=F\left(t_{n}^{2}, u_{n}\left(t_{n}^{1}\right)\right)-\bar{e} u_{n}\left(t_{n}^{1}\right) .
$$

Set

$$
\rho_{n}=\frac{1}{2}\left(\frac{v_{n}\left(\alpha_{n}\right)-\|e\|_{L^{1}}-k T|\bar{e}|}{M_{n}}\right)^{2} .
$$

Then

$$
\lim _{n \rightarrow+\infty} \rho_{n}=0
$$

Indeed, since $M_{n}=u_{n}\left(t_{n}^{1}\right)$, we have

$$
B_{1}\left(\alpha_{n}\right)=F\left(t_{n}^{2}, d\right)-\bar{e} d+\rho_{n} M_{n}^{2} \leq F\left(t_{n}^{2}, u_{n}\left(t_{n}^{1}\right)\right)-\bar{e} u_{n}\left(t_{n}^{1}\right)=F\left(t_{n}^{2}, M_{n}\right)-\bar{e} M_{n} .
$$

From (2.37) we deduce

$$
\lim _{n \rightarrow+\infty} \frac{F\left(t_{n}^{2}, d\right)}{\left(M_{n}\right)^{2}}+\lim _{n \rightarrow+\infty} \rho_{n} \leq \lim _{n \rightarrow+\infty} \frac{F\left(t_{n}^{2}, M_{n}\right)}{\left(M_{n}\right)^{2}} .
$$

Notice that $F\left(t_{n}^{2}, d\right)=\int_{1}^{d} f\left(t_{n}^{2}, s\right) d s$ is bounded, so that

$$
\lim _{n \rightarrow+\infty} \frac{F\left(t_{n}^{2}, d\right)}{\left(M_{n}\right)^{2}}=0
$$

Also, (H2)(i) implies that

$$
\lim _{n \rightarrow+\infty} \frac{F\left(t_{n}^{2}, M_{n}\right)}{\left(M_{n}\right)^{2}}=0
$$

Therefore (2.36) holds; that is, $\lim _{n \rightarrow+\infty} \rho_{n}=0$. Since $\|e\|_{L^{1}}$ and $|\bar{e}|$ are bounded, it follows that

$$
\lim _{n \rightarrow+\infty} \frac{v_{n}\left(\alpha_{n}\right)}{M_{n}}=0
$$

It is clear that (2.41) contradicts (2.17).

Case 2. $f(\cdot, s)$ is decreasing for each fixed $s \in(0,+\infty)$.

In this case we consider the function $B_{2}$ defined by

$$
B_{2}(t)=F\left(\alpha_{n}, u_{n}(t)\right)-\bar{e} u_{n}(t)+\frac{1}{2}\left(v_{n}(t)-\|e\|_{L^{1}}-k T|\bar{e}|\right)^{2} .
$$


Then

$$
\begin{aligned}
B_{2}^{\prime}(t) & =\left(f\left(\alpha_{n}, u_{n}(t)\right)-\bar{e}\right)\left(\int_{\alpha_{n}}^{t}[e(s)-\bar{e}] d s+\|e\|_{L^{1}}+k T|\bar{e}|\right) \\
& +\left(f\left(\alpha_{n}, u_{n}(t)\right)-f\left(t, u_{n}(t)\right)\right)\left(v_{n}(t)-\|e\|_{L^{1}}-k T|\bar{e}|\right) \\
& \geq 0 \quad \forall t \in\left[\alpha_{n}, t_{n}^{2}\right] .
\end{aligned}
$$

Repeating the same reasoning as in Case 1, we arrive at a contradiction.

Therefore, we deduce that there must exist $R>0$ such that $M_{n}=\max _{t \in[0, k T]} u_{n}(t) \leq R$ for each $n$.

Next, we prove that there is $r>0$ such that $u_{n}(t) \geq r$ for every $t \in[0, k T]$. Assuming that this is not true we will obtain a contradiction.

Consider the following sets

$$
\begin{gathered}
I_{1 / n}=\left\{t \in[0, k T] ; u_{n}(t)<\frac{1}{n}\right\}, \\
I_{1 / n, 1 / d}=\left\{t \in[0, k T] ; \frac{1}{n} \leq u_{n}(t)<\frac{1}{d}\right\}, \\
I_{1 / d, R}=\left\{t \in[0, k T] ; \frac{1}{d} \leq u_{n}(t) \leq R\right\} .
\end{gathered}
$$

It is clear that for $n \geq R, u_{n}(t) \leq R \leq n$. Also, we cannot have $u_{n}(t) \geq 1 / n$ for every $t \in[0, k T]$, for otherwise we would have

$$
\frac{1}{n} \leq u_{n}(t) \leq n \quad \forall t \in[0, k T]
$$

which contradicts the assumption $\left\{u_{n}(t) ; t \in \mathbb{R}\right\} \nsubseteq[(1 / n), n]$. Hence, for $n \geq R$ there exists $t_{n}^{3} \in[0, k T]$ such that $u_{n}\left(t_{n}^{3}\right)<1 / n$. This shows that $I_{1 / n} \neq \emptyset$. The continuity of $u_{n}$ implies that $I_{1 / n}$ is open, and so meas $\left(I_{1 / n}\right) \neq 0$.

Define

$$
\Psi_{n}=\int_{0}^{k T}\left[f_{\mu_{n}}\left(t, u_{n}(t)\right)-\bar{e}\right] d t, \quad n \in \mathbb{N}
$$

It follows from (2.9) that $\Psi_{n}=0$. On the other hand

$$
\Psi_{n}=\int_{I_{1 / n}}\left[f_{\mu_{n}}\left(t, u_{n}(t)\right)-\bar{e}\right] d t+\int_{I_{1 / n, 1 / d}}\left[f_{\mu_{n}}\left(t, u_{n}(t)\right)-\bar{e}\right] d t+\int_{I_{1 / d, R}}\left[f_{\mu_{n}}\left(t, u_{n}(t)\right)-\bar{e}\right] d t
$$

(i) Assume we are integrating positively on all subintervals of $[0, k T]$. If $t \in I_{1 / n, 1 / d}$, then $u_{n}(t) \in[1 / n, 1 / d) \subset(0,1 / d)$. So that, by Lemma 2.1,

$$
\int_{I_{1 / n, 1 / d}}\left[f_{\mu_{n}}\left(t, u_{n}(t)\right)-\bar{e}\right] d t<0
$$

For $t \in I_{1 / d, R}$ we have $u_{n}(t) \in[1 / d, R]$. This means that $u_{n}(t)$ is bounded uniformly in $t \in$ $I_{1 / d, R}$. Since $f_{\mu_{n}}$ is continuous it is bounded on $I_{1 / d, R}$. 
Let

$$
c=\max \left\{\left|f_{\mu_{n}}(t, x)\right| ; t \in[0, k T], \frac{1}{d} \leq x \leq R\right\}=\max _{I_{1 / d, R}}\left\{\left|f_{\mu_{n}}(t, x)\right|\right\} .
$$

Then

$$
\left|\int_{I_{1 / d, R}}\left[f_{\mu_{n}}\left(t, u_{n}(t)\right)-\bar{e}\right] d t\right| \leq \int_{I_{1 / d, R}}\left[\left|f_{\mu_{n}}\left(t, u_{n}(t)\right)\right|+|\bar{e}|\right] d t \leq k T(c+|\bar{e}|) .
$$

It follows from $(2.47),(2.48)$, and (2.50) that

$$
\Psi_{n}<\int_{I_{1 / n}}\left[f_{\mu_{n}}\left(t, u_{n}(t)\right)-\bar{e}\right] d t+k T(c+|\bar{e}|)
$$

Claim 3.

$$
\lim _{n \rightarrow \infty} \int_{I_{1 / n}}\left[f_{\mu_{n}}\left(t, u_{n}(t)\right)-\bar{e}\right] d t=-\infty
$$

Proof. Recall that $\mu_{n} \in(0,1 / n)$ and $u_{n}(t)<1 / n$ for each $t \in I_{1 / n}$. Then, if $u_{n}(t)<\mu_{n}$ we have $f_{\mu_{n}}\left(t, u_{n}(t)\right)=f\left(t, \mu_{n}\right)$, and if $u_{n}(t) \in\left[\mu_{n}, 1 / n\right)$, we have $f_{\mu_{n}}\left(t, u_{n}(t)\right)=f\left(t, u_{n}(t)\right)$. In both cases condition (H1)(ii) and the continuity of $f$ imply that $\lim _{n \rightarrow \infty}\left(f_{\mu_{n}}\left(t, u_{n}(t)\right)-\bar{e}\right)=-\infty$ for every $t \in I_{1 / n}$.

Since $\bar{e}$ is bounded, then (2.51) implies that

$$
\lim _{n \rightarrow+\infty} \Psi_{n}=-\infty,
$$

which is a contradiction with (2.9).

(ii) If we integrate negatively on all subintervals of $[0, k T]$ we will obtain $\lim _{n \rightarrow+\infty} \Psi_{n}=$ $+\infty$, which, again, contradicts (2.9). Thus, the proof of Lemma 2.2 is complete.

Remark 2.3. Lemma 2.2 shows that any $k T$-periodic solution $u$ of (2.6), with $\mu=r_{k}$ is a solution of (1.1), since it satisfies $u(t) \geq r_{k}$ for all $t \in \mathbb{R}$ and $f_{r_{k}}(t, u(t))=f(t, u(t))$.

In the remainder of the paper we shall deal with (2.6), with $\mu=r_{k}$ instead of (1.1). Let $F_{r_{k}}(t, u)=\int_{1}^{u} f_{r_{k}}(t, s) d s$ be a primitive of $f_{r_{k}}$ defined for all $t \in I$ and $u \in \mathbb{R}$.

Lemma 2.4. If (H1) and (H2) hold, then $f_{r_{k}}$ and $F_{r_{k}}$ satisfy the following conditions.

(L1) $f_{r_{k}}$ is defined and continuous in $(t, u) \in I \times \mathbb{R}$ and $T$-periodic with respect to $t \in I$.

(L2) $\liminf \operatorname{in|\rightarrow +\infty }_{\mid u} 2 F_{r_{k}}(t, u) / u^{2}=0$, uniformly in $t \in I$.

(L3) $\exists d>1$ such that for $u \in(-\infty, 1 / d) \cup(d,+\infty)$ it holds $\left(f_{r_{k}}(t, u)-\bar{e}\right)(u-1)>0$ uniformly in $t \in I$.

(L4) $\lim _{|u| \rightarrow+\infty} \int_{0}^{T}\left[F_{r_{k}}(t, u)-\bar{e} u\right] d t=+\infty$. 


\subsection{Existence of $k T$-Periodic Solutions for Equation (2.6), $\mu=r_{k}$}

Using a variational method we shall show that equation (2.6), with $\mu=r_{k}$ has infinitely many $k T$-periodic solutions. In fact, we have the following.

Lemma 2.5. Assume that $e$ is locally integrable T-periodic function and the conditions (L1), (L2), (L3), (L4) hold. Then (2.6), with $\mu=r_{k}$ admits a $k T$-periodic solution $u_{k}$.

Proof. We shall rely on a variational method and more precisely on the saddle point theorem.

Define for each $k \geq 1$ the action functional $J_{k}: H_{k T}^{1} \rightarrow \mathbb{R}$ by

$$
J_{k}(u)=\int_{0}^{k T}\left[\frac{1}{2}\left[u^{\prime}(t)\right]^{2}-F_{r_{k}}(t, u(t))+e(t) u(t)\right] d t .
$$

$J_{k}$ is well defined on $H_{k T}^{1}$, weakly lower semicontinuous and continuously differentiable on $H_{k T}^{1}$. Furthermore,

$$
\left\langle J_{k}^{\prime}(u), v\right\rangle=\int_{0}^{k T}\left[u^{\prime}(t) v^{\prime}(t)-f_{r_{k}}(t, u(t)) v(t)+e(t) v(t)\right] d t, \quad \forall u, v \in H_{k T}^{1}
$$

The critical points of $J_{k}$ are precisely the weak solutions of equation (2.6), with $\mu=r_{k}$.

First, we show that the functional $J_{k}$ satisfies the Palais-Smale condition.

For this, let $k \geq 1$ be fixed and let $\left(u_{n}\right)_{n \in \mathbb{N}}$ be a sequence in $H_{k T}^{1}$ such that $\left(J_{k}\left(u_{n}\right)\right)_{n \in \mathbb{N}}$ is bounded and $\lim _{n \rightarrow+\infty} J_{k}^{\prime}\left(u_{n}\right)=0$. Then $\left(u_{n}\right)_{n \in \mathbb{N}}$ has a convergent subsequence.

Suppose, on the contrary, that $\lim _{n \rightarrow+\infty}\left\|u_{n}\right\|_{H_{k T}^{1}}=+\infty$. Condition (L2) implies that for any $\varepsilon>0$, small enough, there is $C_{\varepsilon} \geq 0$ such that

$$
F_{r_{k}}(t, u) \leq \varepsilon u^{2}+C_{\varepsilon}, \quad \forall u \in \mathbb{R} .
$$

Writing $u_{n}(t)=\overline{u_{n}}+\widetilde{u_{n}}(t)$ for $t \in[0, k T]$, we obtain

$$
-\int_{0}^{k T} F_{r_{k}}\left(t, u_{n}(t)\right) d t \geq-\varepsilon k T\left|\overline{u_{n}}\right|^{2}-\varepsilon \int_{0}^{k T}\left|\widetilde{u_{n}}(t)\right|^{2} d t-2 \varepsilon \overline{u_{n}} \int_{0}^{k T} \widetilde{u_{n}}(t) d t-C_{\varepsilon} k T .
$$

Since $\widetilde{u_{n}} \in H^{-}$, we have $\int_{0}^{k T} \widetilde{u_{n}}(t) d t=k T \widetilde{\widetilde{u_{n}}}=0$, so that

$$
-\int_{0}^{k T} F_{r_{k}}\left(t, u_{n}(t)\right) d t \geq-\varepsilon\left\|\widetilde{u_{n}}\right\|_{L^{2}}^{2}-\varepsilon k T\left|\overline{u_{n}}\right|^{2}-C_{\varepsilon} k T .
$$

Now, Hölder's inequality gives

$$
\int_{0}^{k T} e(t) u_{n}(t) d t=\overline{u_{n}} \int_{0}^{k T} e(t) d t+\int_{0}^{k T} e(t) \widetilde{u_{n}}(t) d t \geq-\left|\overline{u_{n}}\right| k T|\bar{e}|-\|e\|_{L^{2}}\left\|\widetilde{u_{n}}\right\|_{L^{2}}
$$


Since $u_{n}^{\prime}(t)=\widetilde{u_{n}}(t)$ for $t \in[0, k T]$, it follows from (2.54), (2.58), and (2.59)

$$
J_{k}\left(u_{n}\right) \geq \frac{1}{2}\left\|\widetilde{u_{n}}\right\|_{L^{2}}^{2}-\varepsilon\left\|\widetilde{u_{n}}\right\|_{L^{2}}^{2}-\varepsilon k T\left|\overline{u_{n}}\right|^{2}-C_{\varepsilon} k T-k T|\bar{e}|\left|\overline{u_{n}}\right|-\|e\|_{L^{2}}\left\|\widetilde{u_{n}}\right\|_{L^{2}} .
$$

Wirtinger's inequality

$$
\left\|\widetilde{u_{n}^{\prime}}\right\|_{L^{2}}^{2} \geq\left(\frac{4 \pi^{2}}{4 \pi^{2}+(k T)^{2}}\right)\left\|\widetilde{u_{n}}\right\|_{H_{k T}^{1}}^{2}
$$

combined with the inequality $\left\|\widetilde{u_{n}}\right\|_{L^{2}} \leq\left\|\widetilde{u_{n}}\right\|_{H_{k T}^{1}}$ give

$$
J_{k}\left(u_{n}\right)+\left(\varepsilon k T\left|\overline{u_{n}}\right|^{2}+k T|\bar{e}|\left|\overline{u_{n}}\right|\right)+C_{\varepsilon} k T \geq\left\|\widetilde{u_{n}}\right\|_{H_{k T}^{1}}\left(\left[\frac{2 \pi^{2}}{4 \pi^{2}+(k T)^{2}}-\varepsilon\right]\left\|\widetilde{u_{n}}\right\|_{H_{k T}^{1}}-\|e\|_{L^{2}}\right) .
$$

This leads to

$$
\lim _{n \rightarrow+\infty}\left|\overline{u_{n}}\right|=+\infty
$$

Indeed, if (2.63) does not hold then there would exist a subsequence of $\left(\overline{u_{n}}\right)_{n \in \mathbb{N}}$, still denoted the same, which is bounded. Since $\left(J_{k}\left(u_{n}\right)\right)_{n \in \mathbb{N}}$ and $\bar{e}$ are bounded and $\varepsilon$ is chosen arbitrarily small, then (2.62) implies that $\left\|\widetilde{u_{n}}\right\|_{H_{k T}^{1}}$ is bounded. It follows from the inequality

$$
\left\|u_{n}\right\|_{H_{k T}^{1}} \leq \sqrt{k T}\left|\overline{u_{n}}\right|+\left\|\widetilde{u_{n}}\right\|_{H_{k T}^{1}}
$$

that $\left\|u_{n}\right\|_{H_{k T}^{1}}$ is bounded, but this contradicts our assumption $\lim _{n \rightarrow+\infty}\left\|u_{n}\right\|_{H_{k T}^{1}}=+\infty$. Therefore, (2.63) holds.

Using Wirtinger's inequality

$$
\left\|\widetilde{u_{n}}\right\|_{L^{2}} \leq \frac{k T}{2 \pi}\left\|\widetilde{u_{n}}\right\|_{L^{2}}
$$

in (2.60), we get

$$
\frac{\left\|\widetilde{u_{n}}\right\|_{L^{2}}}{\left|\overline{u_{n}}\right|}\left(\left[\frac{1}{2}-\varepsilon\left(\frac{k T}{2 \pi}\right)^{2}\right] \frac{\left\|\widetilde{u_{n}}\right\|_{L^{2}}}{\left|\overline{u_{n}}\right|}-\left(\frac{k T}{2 \pi}\right) \frac{\|e\|_{L^{2}}}{\left|\overline{u_{n}}\right|}\right) \leq \frac{J_{k}\left(u_{n}\right)}{\left|\overline{u_{n}}\right|^{2}}+\varepsilon k T+\frac{k T|\bar{e}|}{\left|\overline{u_{n}}\right|}+\frac{C_{\varepsilon} k T}{\left|\overline{u_{n}}\right|^{2}} .
$$

It follows from (2.63) that

$$
\lim _{n \rightarrow+\infty} \frac{\left\|{\widetilde{u_{n}}}^{\prime}\right\|_{L^{2}}}{\left|\overline{u_{n}}\right|}=0
$$


Using Sobolev's inequality we obtain

$$
\lim _{n \rightarrow+\infty} \frac{\left\|\widetilde{u_{n}}\right\|_{\infty}}{\left|\overline{u_{n}}\right|} \leq \sqrt{\frac{k T}{12}} \lim _{n \rightarrow+\infty} \frac{\left\|\widetilde{u_{n}^{\prime}}\right\|_{L^{2}}}{\left|\overline{u_{n}}\right|}=0 .
$$

The identity $u_{n}(t)=\overline{u_{n}}\left(1+\left(\widetilde{u_{n}}(t) / \overline{u_{n}}\right)\right)$ for all $t \in[0, k T]$ and (2.63) imply that

$$
\lim _{n \rightarrow+\infty} \min _{t \in[0, k T]}\left|u_{n}(t)\right|=+\infty
$$

Assume that $\lim _{n \rightarrow+\infty} \min _{t \in[0, k T]} u_{n}(t)=+\infty$ (the other case can be treated similarly). Then for $n$ large enough, $u_{n}(t)>d$ uniformly in $t \in[0, k T]$. By (L3) we have for all $t \in[0, k T]$

$$
f_{r_{k}}\left(t, u_{n}(t)\right)-\bar{e}>0
$$

Consequently, for $n$ large enough

$$
\int_{0}^{k T}\left|f_{r_{k}}\left(t, u_{n}(t)\right)-\bar{e}\right| d t=\int_{0}^{k T}\left[f_{r_{k}}\left(t, u_{n}(t)\right)-\bar{e}\right] d t=\left|\int_{0}^{k T}\left[f_{r_{k}}\left(t, u_{n}(t)\right)-\bar{e}\right] d t\right| .
$$

Since $\lim _{n \rightarrow+\infty} J_{k}^{\prime}\left(u_{n}\right)=0$, then for all $v \in H_{k T}^{1}$ and for $n$ large enough

$$
\left|\int_{0}^{k T}\left[u_{n}^{\prime}(t) v^{\prime}(t)-f_{r_{k}}\left(t, u_{n}(t)\right) v(t)+e(t) v(t)\right] d t\right| \leq \varepsilon_{n}\|v\|_{H_{k T^{\prime}}^{\prime}}
$$

where $\varepsilon_{n}>0$ for every $n$, and $\lim _{n \rightarrow+\infty} \varepsilon_{n}=0$. In particular, if we take $v(t)=-1$ in the above inequality we obtain for every $n \in \mathbb{N}$

$$
\left|\int_{0}^{k T}\left[f_{r_{k}}\left(t, u_{n}(t)\right)-\bar{e}\right] d t\right| \leq \varepsilon_{n} \sqrt{k T}
$$

which infer

$$
\begin{aligned}
\int_{0}^{k T}\left|f_{r_{k}}\left(t, u_{n}(t)\right)\right| d t & \leq \int_{0}^{k T}\left|f_{r_{k}}\left(t, u_{n}(t)\right)-\bar{e}\right| d t+\int_{0}^{k T}|\bar{e}| d t \\
& =\left|\int_{0}^{k T}\left[f_{r_{k}}\left(t, u_{n}(t)\right)-\bar{e}\right] d t\right|+\int_{0}^{k T}|\bar{e}| d t \leq \varepsilon_{n} \sqrt{k T}+k T|\bar{e}|
\end{aligned}
$$

Now, taking $v=\widetilde{u_{n}}$ in (2.72) we obtain

$$
\varepsilon_{n}\left\|\widetilde{u_{n}}\right\|_{H_{k T}^{1}} \geq\left\|\widetilde{u_{n}^{\prime}}\right\|_{L_{2}}^{2}-\left|\int_{0}^{k T}\left[f_{r_{k}}\left(t, u_{n}(t)\right)-e(t)\right] \widetilde{u_{n}}(t) d t\right| .
$$


Obviously, we have for $n$ large enough

$$
\begin{aligned}
\left|\int_{0}^{k T}\left[f_{r_{k}}\left(t, u_{n}(t)\right)-e(t)\right] \widetilde{u_{n}}(t) d t\right| & \leq \sup _{t \in[0, k T]}\left|\widetilde{u_{n}}(t)\right|\left(\int_{0}^{k T}\left|f_{r_{k}}\left(t, u_{n}(t)\right)\right| d t+\int_{0}^{k T}|e(t)| d t\right) \\
& \leq\left\|\widetilde{u_{n}}\right\|_{\infty}\left(\varepsilon_{n} \sqrt{k T}+k T|\bar{e}|+\|e\|_{L^{1}}\right) .
\end{aligned}
$$

Thus, for $n$ large enough, (2.75) implies that

$$
\varepsilon_{n}\left\|\widetilde{u_{n}}\right\|_{H_{k T}^{1}} \geq\left\|\widetilde{u_{n}^{\prime}}\right\|_{L^{2}}^{2}-\left\|\widetilde{u_{n}}\right\|_{\infty}\left(\varepsilon_{n} \sqrt{k T}+k T|\bar{e}|+\|e\|_{L^{1}}\right)
$$

Sobolev's inequality

$$
\left\|\widetilde{u_{n}}\right\|_{\infty} \leq \sqrt{\frac{k T}{12}}\left\|{\widetilde{u_{n}}}^{\prime}\right\|_{L^{2}} \leq \sqrt{\frac{k T}{12}}\left\|\widetilde{u_{n}}\right\|_{H_{k T}^{1}}
$$

and Wirtinger's inequality combined with (2.77) give, for $n$ large enough,

$$
\varepsilon_{n}\left\|\widetilde{u_{n}}\right\|_{H_{k T}^{1}} \geq\left(\frac{4 \pi^{2}}{4 \pi^{2}+(k T)^{2}}\right)\left\|\widetilde{u_{n}}\right\|_{H_{k T}^{1}}^{2}-\sqrt{\frac{k T}{12}}\left\|\widetilde{u_{n}}\right\|_{H_{k T}^{1}}\left(\varepsilon_{n} \sqrt{k T}+k T|\bar{e}|+\|e\|_{L^{1}}\right) .
$$

So, for $n$ large enough, we deduce that

$$
\left\|\widetilde{u_{n}}\right\|_{H_{k T}^{1}} \leq \ell:=\left(\frac{4 \pi^{2}+(k T)^{2}}{4 \pi^{2}}\right)\left(1+\sqrt{\frac{k T}{12}}\left[\sqrt{k T}+k T|\bar{e}|+\|\ell\|_{L^{1}}\right]\right) .
$$

Hence $\left(\widetilde{u_{n}}\right)_{n}$ is bounded in $H_{k T}^{1}$. Consequently $\left\|u_{n}^{\prime}\right\|_{L^{2}}=\left\|\widetilde{u_{n}^{\prime}}\right\|_{L^{2}} \leq\left\|\widetilde{u_{n}}\right\|_{H_{k T}^{1}} \leq \ell$. Since $\left(J_{k}\left(u_{n}\right)\right)_{n \in \mathbb{N}}$ is bounded, it follows that

$$
\int_{0}^{k T}\left[F_{r_{k}}\left(t, u_{n}(t)\right)-e(t) u_{n}(t)\right] d t
$$

is bounded. Hölder's inequality gives

$$
\left|\int_{0}^{k T} e(t) \widetilde{u_{n}}(t) d t\right| \leq\|e\|_{L^{2}}\left\|\widetilde{u_{n}}\right\|_{L^{2}} \leq\|e\|_{L^{2}}\left\|\widetilde{u_{n}}\right\|_{H_{k T}^{1}} \leq \ell\|e\|_{L^{2}}
$$

Since

$$
\int_{0}^{k T} F_{r_{k}}\left(t, u_{n}(t)\right) d t-\overline{u_{n}} \int_{0}^{k T} e(t) d t=\int_{0}^{k T}\left[F_{r_{k}}\left(t, u_{n}(t)\right)-e(t) u_{n}(t)\right] d t+\int_{0}^{k T} e(t) \widetilde{u_{n}}(t) d t,
$$


it follows that

$$
\int_{0}^{k T} F_{r_{k}}\left(t, u_{n}(t)\right) d t-\overline{u_{n}} \int_{0}^{k T} e(t) d t
$$

is bounded. But,

$$
\overline{u_{n}} \int_{0}^{k T} e(t) d t=k T \bar{e} \overline{u_{n}}=k T \bar{e} \frac{1}{k T} \int_{0}^{k T} u_{n}(t) d t=\int_{0}^{k T} \bar{e} u_{n}(t) d t
$$

Consequently, there exists $C>0$ such that

$$
\int_{0}^{k T}\left[F_{r_{k}}\left(t, u_{n}(t)\right)-\bar{e} u_{n}(t)\right] d t \leq C
$$

On the other hand, extending $F_{r_{k}}$ by $T$-periodicity we obtain

$$
\begin{aligned}
\int_{0}^{k T}\left[F_{r_{k}}\left(t, u_{n}(t)\right)-\bar{e} u_{n}(t)\right] d t & =\sum_{j=0}^{k-1} \int_{0}^{T}\left[F_{r_{k}}\left(t+j T, u_{n}(t+j T)\right)-\bar{e} u_{n}(t+j T)\right] d t \\
& =\sum_{j=0}^{k-1} \int_{0}^{T}\left[F_{r_{k}}\left(t, u_{n}(t+j T)\right)-\bar{e} u_{n}(t+j T)\right] d t .
\end{aligned}
$$

Setting $x_{n}=u_{n}(t+j T)$ for $t \in[0, T]$, we get

$$
\int_{0}^{k T}\left[F_{r_{k}}\left(t, u_{n}(t)\right)-\bar{e} u_{n}(t)\right] d t=\sum_{j=0}^{k-1} \int_{0}^{T}\left[F_{r_{k}}\left(t, x_{n}\right)-\bar{e} x_{n}\right] d t=k \int_{0}^{T}\left[F_{r_{k}}\left(t, x_{n}\right)-\bar{e} x_{n}\right] d t
$$

From (2.75) $\left|x_{n}\right|=\left|u_{n}(t+j T)\right| \rightarrow+\infty$ when $n \rightarrow+\infty$ uniformly in $t \in[0, k T]$. By (L4) we have

$$
\lim _{n \rightarrow+\infty} \int_{0}^{k T}\left[F_{r_{k}}\left(t, u_{n}(t)\right)-\bar{e} u_{n}(t)\right] d t=k \lim _{|x| \rightarrow+\infty} \int_{0}^{T}\left[F_{r_{k}}(t, x)-\bar{e} x\right] d t=+\infty
$$

This is a clear contradiction to (2.86). Therefore, $\left(u_{n}\right)_{n \in \mathbb{N}}$ is bounded in $H_{k T}^{1}$, and so it has a convergent subsequence. This shows that $J_{k}$ satisfies the Palais-Smale condition. Next, we show that $J_{k}$ has a geometry of a Saddle. For, let $u \in H^{-}$then we have $u=\widetilde{u_{n}}$ and $\bar{u}=0$, so that

$$
J_{k}\left(\widetilde{u_{n}}\right)=\frac{1}{2}\left\|\widetilde{u_{n}}\right\|_{L^{2}}^{2}-\int_{0}^{k T}\left[F_{r_{k}}\left(t, \widetilde{u_{n}}(t)\right)-e(t) \widetilde{u_{n}}(t)\right] d t
$$


Proceeding as before, we get an inequality similar to (2.72) by replacing $u_{n}$ by $\widetilde{u_{n}}$ and $\bar{u}_{n}$ by 0 ,

$$
J_{k}\left(\widetilde{u_{n}}\right) \geq\left\|\widetilde{u_{n}}\right\|_{H_{k T}^{1}}\left(\left[\frac{2 \pi^{2}}{4 \pi^{2}+(k T)^{2}}-\varepsilon\right]\left\|\widetilde{u_{n}}\right\|_{H_{k T}^{1}}-\|e\|_{L^{2}}\right)-C_{\varepsilon} k T .
$$

Since $\varepsilon$ is chosen arbitrary small, we obtain

$$
\lim _{\left\|\widetilde{u}_{n}\right\|_{H_{k T}^{1}} \rightarrow+\infty} J_{k}\left(\widetilde{u_{n}}\right)=+\infty
$$

which shows that $J_{k}$ is coercive. Hence, $J_{k}$ admits a bounded minimizing sequence. Furthermore, $J_{k}$ is weakly lower semicontinuous on $H_{k T}^{1}$, then

$$
\inf _{H^{-}} J_{k}>-\infty
$$

For $s$, a constant function, we have $\|s\|_{H_{k T}^{1}}=|s| \sqrt{k T} \rightarrow+\infty$ if and only if $|s| \rightarrow+\infty$. Then

$$
J_{k}(s)=-\int_{0}^{k T}\left[F_{r_{k}}(t, s)-e(t) s\right] d t
$$

Extending $F_{r_{k}}$ by $T$-periodicity we obtain

$$
\begin{aligned}
J_{k}(s) & =-\int_{0}^{k T}\left[F_{r_{k}}(t, s)-\bar{e} s\right] d t=-\sum_{i=0}^{k-1} \int_{0}^{T}\left[F_{r_{k}}(t+i T, s)-\bar{e} s\right] d t s \\
& =-k \int_{0}^{T}\left[F_{r_{k}}(t, s)-\bar{e} s\right] d t .
\end{aligned}
$$

Condition (L4) implies that

$$
\lim _{|s| \rightarrow+\infty}\left[-J_{k}(s)\right]=k \lim _{|s| \rightarrow+\infty} \int_{0}^{T}\left[F_{r_{k}}(t, s)-\bar{e} s\right] d t=+\infty
$$

Hence, for each $k \geq 1,\left(-J_{k}\right)$ is coercive on the space of constant functions. Then for each $k \geq 1$, there exists $\eta_{k}>0$, large enough, such that

$$
J_{k}\left(\eta_{k}\right) \rightarrow-\infty, \quad \text { and } J_{k}\left(-\eta_{k}\right) \rightarrow-\infty
$$

Thus,

$$
\max \left(J_{k}\left(-\eta_{k}\right), J_{k}\left(\eta_{k}\right)\right) \rightarrow-\infty \quad \text { when } \eta_{k} \rightarrow+\infty
$$


Therefore,

$$
\max \left(J_{k}\left(-\eta_{k}\right), J_{k}\left(\eta_{k}\right)\right)<\inf _{H^{-}} J_{k}
$$

Let $I_{\eta_{k}}$ be the open interval of $\mathbb{R}$ centered at 0 and with radius $\eta_{k}$. Since $H^{+}=\mathbb{R}$ it is clear that

$$
\partial I_{\eta_{k}} \cap H^{+}=\left\{-\eta_{k}, \eta_{k}\right\}
$$

Therefore, we have

$$
\max _{\partial I_{\eta_{k}} \cap H^{+}} J_{k}<\inf _{H^{-}} J_{k}
$$

with $H^{+} \oplus H^{-}=H_{k T}^{1}$. Thus $J_{k}$ has a geometry of Saddle.

Finally, all conditions of the Saddle point theorem are satisfied. Then for each $k \geq 1, J_{k}$ admits a critical point $\beta_{k}$, which is characterized by

$$
J_{k}\left(\beta_{k}\right)=\inf _{\psi \in \Gamma_{k}} \max _{s \in\left[-\eta_{k}, \eta_{k}\right]} J_{k}(\psi(s))
$$

where

$$
\Gamma_{k}=\left\{\psi \in C\left(\left[-\eta_{k}, \eta_{k}\right], H_{k T}^{1}\right) ; \psi\left(-\eta_{k}\right)=-\eta_{k}, \psi\left(\eta_{k}\right)=\eta_{k}\right\}
$$

Thus for each $k \geq 1, \beta_{k}$ is a weak $k T$-periodic solution of (2.6) with $\mu=r_{k}$ If, furthermore, $e$ is assumed continuous, then $\beta_{k}$ is a classical solution of (2.6) with $\mu=r_{k}$.

This completes the proof of Lemma 2.5.

Remark 2.6. As a consequence of Lemmas 2.2, 2.5 and the Remark 2.3, we conclude that if $(H 1)$ and (H2) are satisfied, then (1.1) admits a sequence $\left(u_{k}\right)_{k \geq 1}$ of $k T$-periodic solutions.

\subsection{Existence of Distinct Subharmonic Solutions for (2.6) with $\mu=r_{k}$}

Note that $J_{k}=J_{m}$ on $H_{k T}^{1} \cap H_{m T}^{1}$ for each $k$ and $m$. This justifies the following definition (see [10, Definition 2.1 page 653]).

Definition 2.7. The level of $u \in \cup_{k} H_{k T}^{1}$ is defined by $J_{m}(u)$ when $u \in H_{m T}^{1}$.

Every functional $J_{k}$ admits at least a critical level which is given by $\beta_{k}=\min _{H_{k T}^{1}} J_{k}$.

Note that nondistinct subharmonic solutions have the same level. Then we deduce that in order to find the multiplicity of distinct subharmonic solutions, we have to search the multiple critical levels. The sequence $\left(\beta_{k}\right)_{k \geq 1}$ is not always increasing, which means that there exists $m \in \mathbb{N}$ such that if $k \in m \mathbb{N}$ then $\beta_{k} \leq \beta_{m}$.

If $\beta_{k}<\beta_{1}$ for an integer $k$, then $\beta_{k}$ is not a level for the $T$-periodic functions and every global minimum of $J_{k}$ is in fact a subharmonic solution of (2.6). 
In our case $J_{k}\left(u_{k}\right)$ is not necessary a global minimum for $J_{k}$ and then even if the condition above is verified, it is still insufficient to deduce the existence of true subharmonic solutions. This is why we prove also that the amplitudes and the minimal periods tend to infinity.

Lemma 2.8. The minimal periods of the solutions $u_{k}$ of (2.6), with $\mu=r_{k}$ tend to infinity.

Proof. Let $u_{k}$ be a weak solution of (2.6) with $\mu=r_{k}$ Then $u_{k}$ is a critical point of $J_{k}$. We show that

$$
\lim _{k \rightarrow+\infty} \frac{1}{k} J_{k}\left(u_{k}\right)=-\infty
$$

Let $\eta_{k} \geq k$ and let $\varphi_{k} \in \Gamma_{k}$ be defined for all $s \in\left[-\eta_{k}, \eta_{k}\right]$ and for all $t \in[0, k T]$ by

$$
\left(\varphi_{k}(s)\right)(t)=s+\left(\eta_{k}-|s|\right) .
$$

We have $\left(\varphi_{k}\left(\eta_{k}\right)\right)(t)=\eta_{k}$ and $\left(\varphi_{k}\left(-\eta_{k}\right)\right)(t)=-\eta_{k}$ for all $t \in[0, k T] .\left(\varphi_{k}(s)\right)(\cdot)$ is constant with respect to $t$ for all $s \in\left[-\eta_{k}, \eta_{k}\right]$ and $\varphi_{k}(s) \in H_{k T}^{1}$. Let $s_{k} \in\left[-\eta_{k}, \eta_{k}\right]$ be such that

$$
J_{k}\left(\varphi_{k}\left(s_{k}\right)\right)=\max _{s \in\left[-\eta_{k}, \eta_{k}\right]} J_{k}\left(\varphi_{k}(s)\right) .
$$

We have

$$
J_{k}\left(u_{k}\right) \leq J_{k}\left(\varphi_{k}\left(s_{k}\right)\right)
$$

Since $\left(\varphi_{k}\left(s_{k}\right)\right)^{\prime}(t)=0$ for all $t \in[0, k T],(2.54)$ implies that

$$
J_{k}\left(\varphi_{k}\left(s_{k}\right)\right)=-\int_{0}^{k T}\left[F_{r_{k}}\left(t,\left(\varphi_{k}\left(s_{k}\right)\right)(t)\right)-e(t)\left(\varphi_{k}\left(s_{k}\right)\right)(t)\right] d t
$$

Extending $F_{r_{k}}$ by $T$-periodicity, we obtain for $k \geq 2$

$$
\begin{aligned}
\frac{1}{k} J_{k}\left(u_{k}\right) \leq \frac{1}{k} J_{k}\left(\varphi_{k}\left(s_{k}\right)\right) & =-\frac{1}{k} \sum_{j=0}^{k-1} \int_{0}^{k T}\left[F_{r_{k}}\left(t+j T,\left(\varphi_{k}\left(s_{k}\right)\right)(t+j T)\right)-\bar{e}\left(\varphi_{k}\left(s_{k}\right)\right)(t+j T)\right] d t s \\
& =-\int_{0}^{T}\left[F_{r_{k}}\left(t,\left(\varphi_{k}\left(s_{k}\right)\right)(t)\right)-\bar{e}\left(\varphi_{k}\left(s_{k}\right)\right)(t)\right] d t
\end{aligned}
$$

We have $\lim _{k \rightarrow+\infty}\left|\left(\varphi_{k}\left(s_{k}\right)\right)(t)\right|=+\infty$ for all $t \in[0, k T]$. Apply (L4) with $u=\left(\varphi_{k}\left(s_{k}\right)\right)(t)$ to obtain

$$
\lim _{k \rightarrow+\infty} \frac{1}{k} J_{k}\left(u_{k}\right) \leq-\lim _{|u| \rightarrow+\infty} \int_{0}^{T}\left[F_{r_{k}}(t, u)-\bar{e} u\right] d t=-\infty
$$

Hence (2.104) holds. 
Now, assume by contradiction that we can extract from the sequence $\left(u_{k}\right)_{k \geq 1}$, of solutions of (2.6) with $\mu=r_{k}$ a subsequence whose minimal periods are bounded. Then for this subsequence we can find a common period $k_{0} T$. The sequence $\left(u_{n}\right)_{n \geq 1}$ of the critical points of $J_{k_{0}}$ satisfies

$$
J_{k_{0}}\left(u_{n}\right)=\frac{1}{n} J_{n}\left(u_{n}\right)
$$

Assuming $\lim _{n \rightarrow+\infty}\left\|u_{n}\right\|_{\infty}=+\infty$ and proceeding as before we arrive at the conclusion $\left(J_{k_{0}}\left(u_{n}\right)\right)_{n}$ is bounded and this contradicts (2.104). This completes the proof of Lemma 2.8.

Lemma 2.9. The amplitudes $A_{k}:=\left(\max _{[0, k T]} u_{k}-\min _{[0, k T]} u_{k}\right)$ of the the solutions $u_{k}$ of equation (2.6) with $\mu=r_{k}$ tend to infinity.

Proof. We have to show that $\lim _{k \rightarrow+\infty} A_{k}=+\infty$. First, we have

$$
\lim _{k \rightarrow+\infty}\left\|u_{k}\right\|_{\infty}=+\infty
$$

Otherwise, we can extract from $\left(u_{k}\right)_{k>1}$ a subsequence converging to some $u^{*}$ with period $n_{0} T$, for some $n_{0}>0$. But, this would contradict Lemma 2.8. Next, we must prove that $\lim _{k \rightarrow+\infty}\left\|\tilde{u}_{k}\right\|_{\infty}=+\infty$. Assume, on the contrary, that $\left\|\tilde{u}_{k}\right\|_{\infty}$ is bounded. If we suppose further that $\left(\left|\tilde{u}_{k}\right|\right)_{k \geq 1}$ is bounded, then $\left\|u_{k}\right\|_{\infty}$ would be bounded and this contradicts (2.112). Hence there exists a subsequence of $\left(u_{k}\right)_{k \geq 1}$, which we label the same, such that $\lim _{k \rightarrow+\infty}\left|\bar{u}_{k}\right|=+\infty$. This implies $\lim _{k \rightarrow+\infty} \min _{[0, k T]}\left|u_{k}(t)\right|=+\infty$. Then, for $k$ sufficiently large and each $j=$ $0,1, \ldots, k-1, u_{k}(t+j T)>d$, uniformly in $t \in(0, T)$. It follows from (L3) that for $k$ large enough $f_{r_{k}}\left(t, u_{k}(t+j T)\right)-\bar{e}>0$, uniformly in $t \in(0, T)$. Equation (2.6) with $\mu=r_{k}$ and the $T$ periodicity of $f_{r_{k}}$ with respect to $t$ give

$$
\int_{0}^{T}\left[\frac{1}{k} \sum_{j=0}^{k-1} f_{r_{k}}\left(t, u_{k}(t+j T)\right)-\bar{e}\right] d t=0
$$

Hence we can use Fatou's Lemma to obtain

$$
0=\int_{0}^{T} \liminf _{k \rightarrow+\infty}\left[\frac{1}{k} \sum_{i=0}^{k-1} f_{r_{k}}\left(t, u_{k}(t+i T)\right)-\bar{e}\right] d t=\int_{0}^{T} \liminf _{x \rightarrow+\infty}\left[f_{r_{k}}(t, x)-\bar{e}\right] d t>0
$$

This is a contradiction. Hence $\lim _{k \rightarrow+\infty}\left\|\tilde{u}_{k}\right\|_{\infty}=+\infty$, and the proof of Lemma 2.9 is complete.

From the above auxiliary results we deduce that equation (2.6) with $\mu=r_{k}$, and consequently (1.1), admits a sequence $\left(u_{k}\right)_{k \geq 1}$ of distinct $k T$-periodic solutions whose amplitudes and minimal periods tend to infinity. Thus, the proof of Theorem 1.1 is complete. 


\section{Acknowledgments}

The authors are grateful to an anonymous referee for suggestions and comments that led to the improvement of the paper. A. Boucherif is grateful to King Fahd University of Petroleum and Minerals for its constant support.

\section{References}

[1] J. Chu and J. J. Nieto, "Recent existence results for second-order singular periodic differential equations," Boundary Value Problems, vol. 2009, Article ID 540863, 20 pages, 2009.

[2] A. Boucherif and N. Daoudi-Merzagui, "Periodic solutions of singular nonautonomous second order differential equations," Nonlinear Differential Equations and Applications, vol. 15, no. 1-2, pp. 147-158, 2008.

[3] N. Daoudi-Merzagui, "Periodic solutions of nonautonomous second order differential equations with a singularity," Applicable Analysis, vol. 73, no. 3-4, pp. 449-462, 1999.

[4] X. Li and Z. Zhang, "Periodic solutions for second-order differential equations with a singular nonlinearity," Nonlinear Analysis, vol. 69, no. 11, pp. 3866-3876, 2008.

[5] J. Mawhin and M. Willem, Critical Point Theory and Hamiltonian Systems, vol. 74 of Applied Mathematical Sciences, Springer, New York, NY, USA, 1989.

[6] P. H. Rabinowitz, Minimax Methods in Critical Point Theory with Applications to Differential Equations, vol. 65 of CBMS Regional Conference Series in Mathematics, American Mathematical Society, Providence, RI, USA, 1986.

[7] A. Fonda, Periodic Solutions of Scalar Second Order Differential Equations with a Singularity, Académie Royale de Belgique, Brussels, Belgium, 1993.

[8] A. Fonda, R. Manásevich, and F. Zanolin, "Subharmonic solutions for some second-order differential equations with singularities," SIAM Journal on Mathematical Analysis, vol. 24, no. 5, pp. 1294-1311, 1993.

[9] A. Fonda and M. Ramos, "Large-amplitude subharmonic oscillations for scalar second-order differential equations with asymmetric nonlinearities," Journal of Differential Equations, vol. 109, no. 2, pp. 354-372, 1994.

[10] E. Serra, M. Tarallo, and S. Terracini, "Subharmonic solutions to second-order differential equations with periodic nonlinearities," Nonlinear Analysis, vol. 41, pp. 649-667, 2000.

[11] C.-L. Tang, "Periodic solutions for nonautonomous second order systems with sublinear nonlinearity," Proceedings of the American Mathematical Society, vol. 126, no. 11, pp. 3263-3270, 1998.

[12] J. Yu, "Subharmonic solutions with prescribed minimal period of a class of nonautonomous Hamiltonian systems," Journal of Dynamics and Differential Equations, vol. 20, no. 4, pp. 787-796, 2008.

[13] X. Zhang and X. Tang, "Subharmonic solutions for a class of non-quadratic second order Hamiltonian systems," Nonlinear Analysis: Real World Applications, vol. 13, no. 1, pp. 113-130, 2012.

[14] L. D. Humphreys, P. J. McKenna, and K. M. O’Neill, "High frequency shaking induced by low frequency forcing: periodic oscillations in a spring-cable system," Nonlinear Analysis. Real World Applications, vol. 11, no. 5, pp. 4312-4325, 2010.

[15] P. Omari, G. Villari, and F. Zanolin, "Periodic solutions of the Liénard equation with one-sided growth restrictions," Journal of Differential Equations, vol. 67, no. 2, pp. 278-293, 1987. 


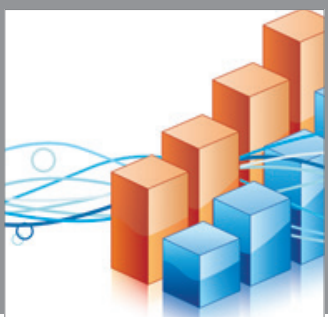

Advances in

Operations Research

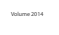

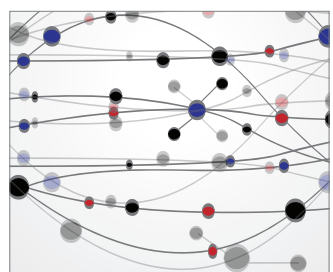

\section{The Scientific} World Journal
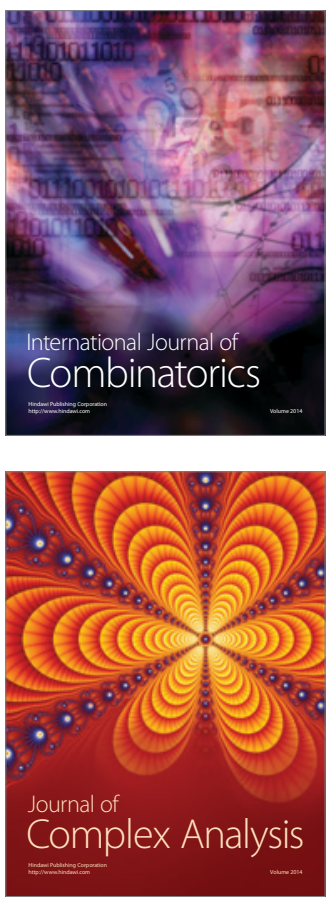

International Journal of

Mathematics and

Mathematical

Sciences
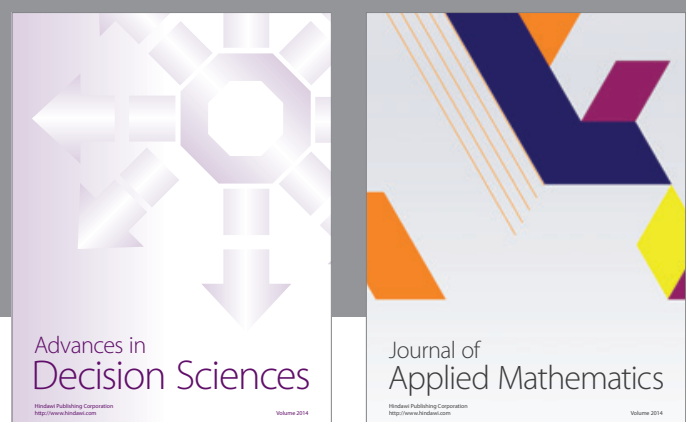

Journal of

Applied Mathematics
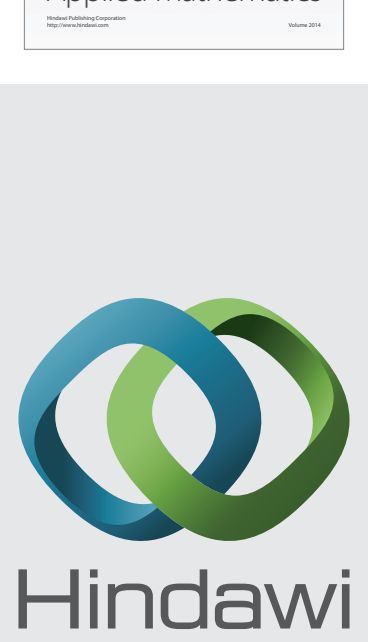

Submit your manuscripts at http://www.hindawi.com
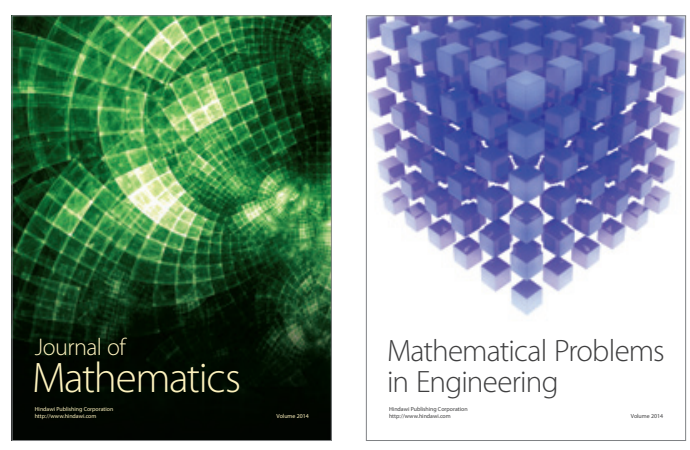

Mathematical Problems in Engineering
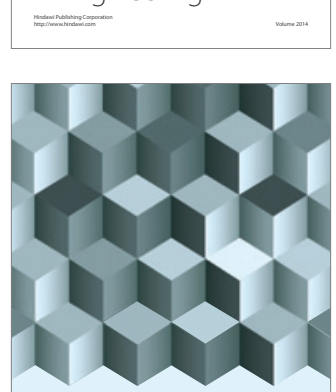

Journal of

Function Spaces
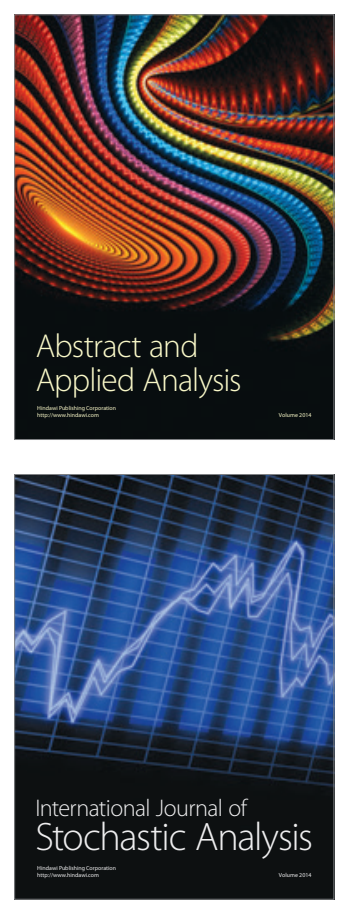

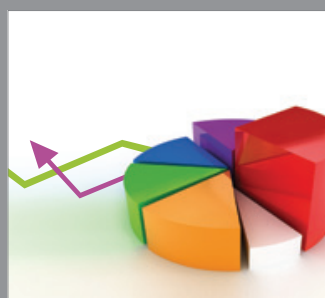

ournal of

Probability and Statistics

Promensencen
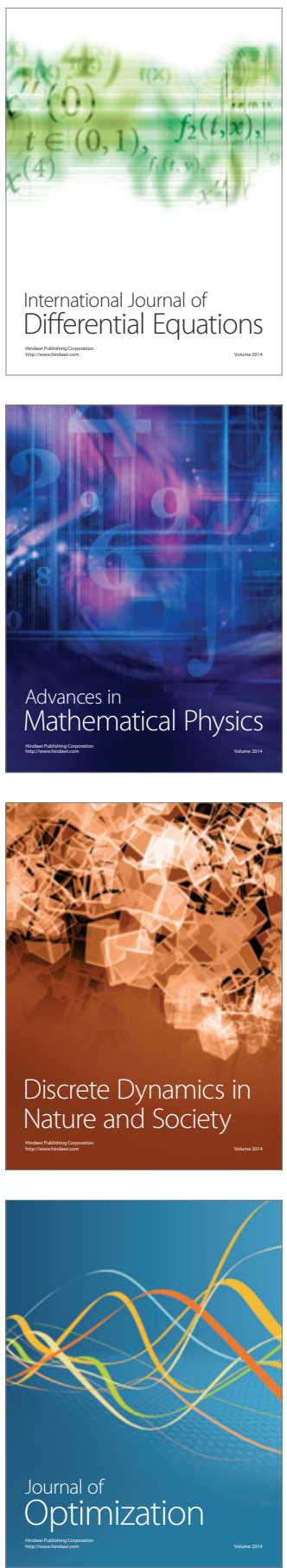